\title{
Performance Evaluation of Machine Learning Techniques using Software Cost Drivers
}

\author{
Manas Gaur \\ Department of Computer Engineering, Delhi Technological University \\ Delhi, India
}

\begin{abstract}
There is a tremendous rise in cost of software, used in organizations. The cost of software ranges from hundred thousand to millions of dollars. The prediction of the software cost beforehand is the challenging area as the rough estimates and the actual cost varies with large differences. The traditional methods are being used since birth of software engineering. These methods based on current project needs, defines the cost based on appropriate weights assigned to scale factors and cost drivers. Application of artificial intelligence in software project planning has given a new methodology for Software Cost Estimation (SCE) that has improved, prediction accuracy. This methodology named Machine Learning Techniques (MLTs) lays emphasis on, similarity to past projects and correlation in the data (training data).Our research work has considered 10 projects along with their costs based on the cost drivers. Using Machine Learning Techniques (MLTs), the research tries to predict the cost, based on the cost drivers. The performance of MLTs was analyzed using root means square error and squared error.
\end{abstract}

\section{Keywords}

Fuzzy Logic, Decision Tree, NNPO, Association Rules, Linear Regression, Perceptron, Naïve Bayes, Neural Network

\section{INTRODUCTION}

The global software market was estimated to be $\$ 120$ billion in 1990, $\$ 151.2$ billion in 1998 , and $\$ 240$ billion in 2000 and is expected to be $\$ 300$ billion by 2014 . Such tremendous rise is unquestionably due to decrease in the cost of hardware resources which forces the software industry to design million dollar software. Another feature that forces the rise in the software development is the low cost of personnel in software industry. US has seen rise of about $19 \%$ in the software cost for four consecutive years. To effectively design software we need accurate and precise estimation of the software resources. Software engineering has laid down principles for effective software development that provides high quality software within budget [7]. Software engineering inculcates software project planning, software risk management, software estimation and software quality. The estimate the software cost, there are few software cost components that have to address precisely such as:-Hardware and software cost, Travel and Training cost, Effort cost (the dominant factor in S/w projects), Salaries of the engineer involved in the project, Social and insurance cost., Effort cost must take overhead into account, Cost of building, Cost of networking and communication, Cost of shared facilities.

Along with the software cost components there are some software pricing factors which must taken into consideration before estimation such as: Market Opportunity, Cost estimate Uncertainty, Non-linear relationship between cost estimation parameters, Technology advancement, High impact due to scale factors and cost drivers, Contractual terms, Requirement Volatility, Financial Health [7]. The algorithmic model used in the software engineering provide an economical approach to software cost estimation but suffers from weaknesses such as high variation in the cost estimation this may pose problems for the manager to estimate the resources precisely, models are based on early measurements therefore do not take into account recent advancement in technology like programming, new algorithms, new principle and research in software engineering, high dependence on cost drivers, human bias and less concern with project developed in past. Machine learning is a concept of artificial intelligence applied to the field of software engineering to enhance the estimation process of the software [5]. In machine learning trends in software project and correlation in data is identified, identification of important attributes that majorly affect the software cost, normalization to enhance investigation area are few methodologies used in the machine learning paradigm. Further discussion in this research covers machine learning techniques along the process flow chart and comparison of the performance result.

\section{LITERATURE SURVEY}

Malhotra [5] analyzed machine learning methods in order to develop models to predict software development effort. The results proved that linear regression MSP and M5rules are effective methods for predicting software development effort. Venkatachalam [1] stated in his research that an artificial neural network approach can be used to model the software cost estimation expertise and results were compared with the COCOMO model. Iman Attarzadeh [6] proposed Fuzzy logic COCOMO II model. Evaluation of the model was carried out in term of Mean of Magnitude of Relative Error (MMRE) and Prediction (PRED). FL-COCOMO II showed $8.03 \%$ improvement in terms of estimation accuracy using MMRE when compared with COCOMO. Using fuzzy logic accuracy in estimation, understandability can be improved which improves software estimates. Efi Papatheocharous [12] address the issue of software cost estimation using an approach to modeling and prediction using artificial neural network (ANN) and input sensitivity analysis (ISA). The validation process includes only highly ranked attributes. The accuracy of the model was same as that of neural network. ANN and ISA is an effective approach for those software projects where subset of selected factors didn't compromise the accuracy of the predicted attribute values. Bill Samson [2] discussed that software cost modeling activity is a hit and try activity where statistical method results in low prediction accuracy. Some experiments were carried out using neural network CMAC or albus perceptron highlighting some of the problems that arise when MLTs are applied in SCE. Experimental results were compared with conventional regression analysis; improved accuracy of predicted values is possible. Bilge Baskeles [11] evaluated the machine learning models against the public data set and found out that use of any one model cannot always yields out best results. 


\section{PROBLEM LITERATURE}

\subsection{Machine Learning Techniques}

Machine Learning Techniques (MLT) or soft computing is a concept of artificial intelligence that perfectly blends with software engineering estimation process [4]. Machine learning system efficiently learns how to estimate from training data of the completed projects. There are many advantages of Machine learning process, to name a few:

- $\quad$ Adaptive Learning

- Self Organization

- Real time operation

- Fault tolerance via redundant information coding

- $\quad$ No expert is required

- No maintenance is required

- Simplicity of input variables

- $\quad$ Easier to depict and understand

Machine learning took over traditional SCE approaches due to deficiency in estimation made by traditional algorithmic models such as:

- A slow adaptation to rapidly changing business requirements

- A tendency to be over budget

- A tendency to be behind schedule

- Difficult to create a complete set of requirements up front.

- Heavy documentation

- Extensive planning

- Rigorous reuse

The machine learning techniques discussed and compared as a part of this research are: Neural Networks(NN), Neural Network with Parameter Optimization(NNPO), Fuzzy Logic trained Neural Network(FLNN), Decision Tree(DT), Linear Regression(LR), Perceptron and Association rules to find pattern in the software cost. [4] Association rules in SCE are not concerned with performance analysis, on the contrary it is used to find correlation and frequent pattern in software cost matrix.

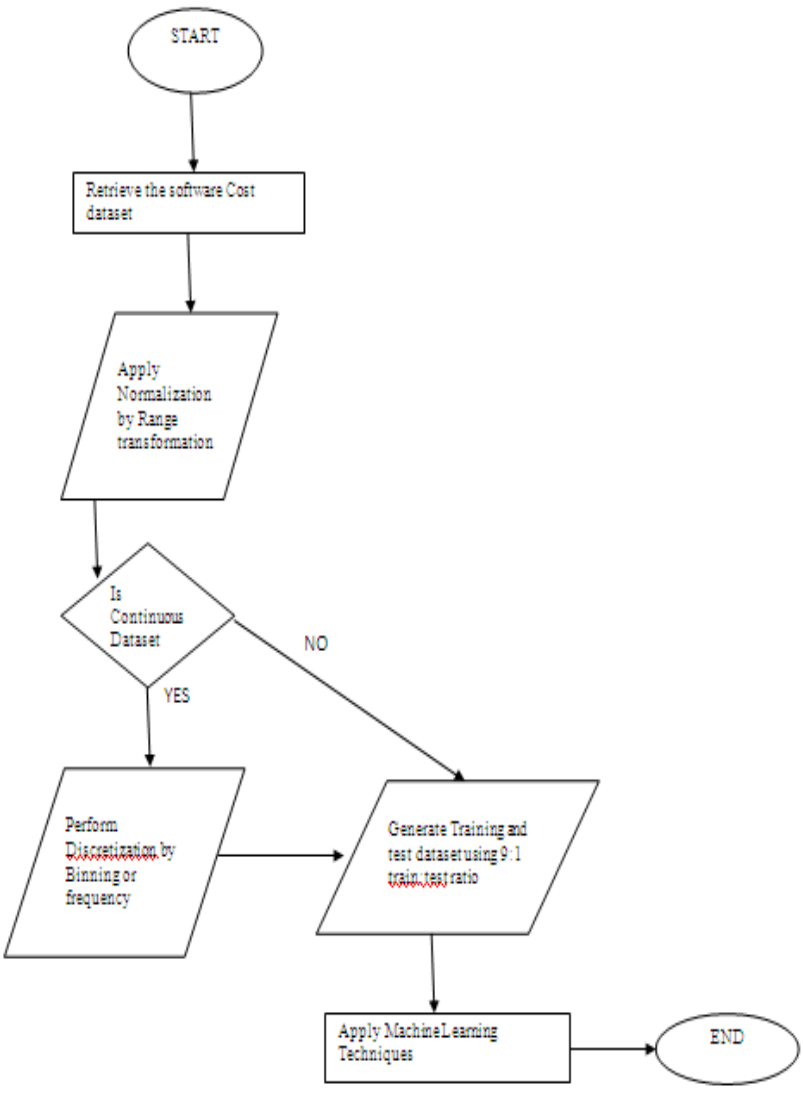

Fig 1: Procedure for applying MLTs on Software Cost Dataset

\subsubsection{Neural Network (NN)}

The neural network trained using back propagation algorithm. The NN is composed of layer which contains neurons. The first layer is the input layer consisting of input variables, the output layer constitutes the output variables and the relation between the two is maintained by the hidden neurons which play the role of detecting high level features and generalization. The back propagation in $\mathrm{NN}$ is a recursive algorithm to improve the learning capability of $\mathrm{NN}$. The sigmoid function is a transfer function used in $\mathrm{NN}$ for classification problems [1]. The sigmoid ("S") curve categorizes the input data into low, normal and high values. The sigmoid function is a special case of logistic function and represented by the equation: $\quad F(I)=1 /\left(1+e^{-I}\right)$ (5)

Where $\mathrm{I}$ is the internal activation.

$$
\Delta w j i(n)=\alpha \Delta w j i(n-1)+\mu \delta j(n) y i(n)
$$

$\Delta \mathrm{w}_{\mathrm{ji}}(\mathrm{n})$ refers the change in weight, which is a dependent variable on sum of $\alpha$ times previous weights and product of learning rate $\mu$, error term $\delta_{j}(n)$ and the input term $\mathrm{y}_{\mathrm{i}}(\mathrm{n})$.

\subsubsection{Neural Network with Parameter Optimization (NNPO)}

NNPO is a process of the modifying the results obtained through neural net. In the parameter optimization process we optimize the parameters of main concern in the NN. In our process we took learning rate and learning momentum a two main parameter to be optimized as they majorly affect the $\mathrm{NN}$ 
working through back propagation.[8] Learning rate is defined a NN training parameter that controls the size of the weights and bias changes during learning. It is defined in the range of $(0,1)$. Learning Momentum simply adds a fraction of previous weights to the current one. It is defined to prevent the system from converging to local minimum or a saddle point. A high value of momentum can overshoot the training process but might decrease the convergence time, a low value can slow down the training process. The value of momentum is defined in the range $(0,1)$. In our research process, while optimizing the learning rate and momentum we obtained 121(11 for Learning rate and 11 for learning momentum) permutation that would run for 500 training cycles. [8] The output showed a significant reduction in the error as compared to NN. For larger projects the change was tremendous of the order of 1.2 to 2.4 points of error difference. The important parameters that can be optimized to improve $\mathrm{NN}$ results are: Hidden layers, Training Set (last column should be the data to be predicted), Target set (that data to be predicted) and Epochs (the number of training set data to be used in back propagation).

\subsubsection{Fuzzy logic trained Neural Network (FLNN):}

Fuzzy logic technique is a superset of predicate logic which states that there is no clear rule to demarcate the data as valid and invalid. Software cost estimation data is ambiguous, vague, obscure and highly sensitive to cost drivers and scale factors [3.] The data is so complex, even $\mathrm{K}$ means produce incorrect clusters. Fuzzy logic with its powerful linguistic representation using membership functions, fuzzy clustering, can signify imprecision in input and output thereby providing more expert knowledge about the variation and correlation in data [6]. The entire code to train NN using fuzzy rules was carried out in MATLAB. We defined sugeno rules for the input variable using Gaussian membership function based on cluster centers derived using sub clustering in matlab. The output variables were made functions of linear type. The fuzzy inference rules generated are used to train the neural network and significant change in the error was observed showing improvement in results of FLNN as compared to NN.

\subsubsection{Decision Tree}

Decision Tree learning uses a decision tree as a procedure model which maps observation about an item to conclusions about the item's target value. In these tree structures, leaves represent class labels and branches represent conjunctions of features that lead to those class labels. Decision tree can be made work top-down or bottom-up [4]. Decision tree can be used to determine gini impurity, information gain from the input data set. Decision tree can be used to derive performance correlation in the dataset. Gini Impurity is a measure of how often a randomly chosen element from the set would be incorrectly labeled if it were randomly labeled according to the distribution of the labels in the subset.

$G . I(f)=\sum_{\mathrm{i}=1}^{\mathrm{m}} \mathrm{fi}(1-\mathrm{fi})=1-\sum_{\mathrm{i}=1}^{\mathrm{m}} \mathrm{fi}^{2}$

Information gain is defined as the entropy from information theory. An entropy typically changes when we use a node in the decision tree to partition the training instances into smaller subsets. A measure of change in entropy is information gain.

I. $G(f)=\sum_{i=1}^{m} \log _{2} f i$

The performance analysis of the decision tree is based on the information gain derived for this research work. The result shows significant improvement over earlier used MLTs. The flow chart shows the flow of work carried out to derive the results

\subsubsection{Nä̈ve Bayes}

Bayesian classifiers are statistical classifiers. They can predict class membership probabilities, such as the probability that a given sample belongs to a particular class. Bayesian classifier is based on Bayes's theorem. Naïve Bayesian classifiers assume that the effect of an attribute value on a given class is independent of the values of the other attributes [9]. This assumption is called class conditional independence. It is made to simplify the computation involved and, in this sense, is considered "naïve". The use of Laplace correction to prevent high influence of zero probabilities [10]. The classification generated using naive bayes have shown significant improvement over the classification results of NN. It has also shown high accuracy in classification results for large software project's datasets.

$$
\text { Bayes probability } P(A i \mid B)=\frac{P(B \mid A i) \cdot P(A i)}{\sum_{j} P(B \mid A j) \cdot P(A j)}
$$

\subsubsection{Linear Regression}

Regression is a machine learning technique used to fit an equation to a dataset. Using,

$$
y=m x+b
$$

Appropriate values for $\mathrm{m}$ and $\mathrm{b}$ to predict the values of $\mathrm{y}$ based on given values of $x$. For our research we take $y=c o s t$ and $x=$ cost drivers. Since work of software cost estimation contains large values therefore modifying the regression equation as [5]

$$
\sum_{i=1}^{m} y i=m * \sum_{i=1}^{m} x i+b
$$

Where yi (S/W cost in dollars) is the target sets column with $\mathrm{i}$ attributes and xi (cost drivers) is the train sets column with $\mathrm{i}$ attributes. LR technique can also generate weights to show the dependency of input attributes on the output attribute.

\subsubsection{Association Rules for Software Size Estimation}

Associative Models are being used to find patterns in large projects containing the nominal data. The 2 main capabilities of associative model are: firstly, the predictive use of association rules, secondly, management of quantitative attributes [4]. The aim is to induce class association rules that allow predicting software size from attributes obtained in early stages of the project. In this application area, most of the attributes are continuous; therefore, they should be discretized before generating the rules $[4,6]$. Discretization is a data mining pre processing task having a special importance in association rule mining since it has a significant influence on the quality and the predictive precision of the induced rules [4].

\subsubsection{Software cost estimation using Perceptron}

One of the goals of Artificial Intelligence is to device system that exhibits adaptive learning. Rosenblatt's perceptron models a human neuron by taking a number of weighted inputs (cost drivers), summing them and giving an output of 1 if the sum is greater than some threshold value and a 0 otherwise [2]. Since a perceptron can be 'trained' by presenting it with training set of input/output pairs and adjusting the weights of the input until the desired output is achieved for every pair in the training set. This process This process is just the statistical one of finding a linear 
discriminant. [2] It is the simplest kind of feed forward network. It takes training set as input and improves the example set using rounds and learning rate which servers as its parameter. It helps in training $\mathrm{NN}$ by providing optimize weights to inputs.

\section{MODEL DESCRIPTION}

Input the Data set containing the cost driver and their values for the past projects and the cost drivers with their values for the present project. Our target output is the prediction of the cost prices using the method of cross validation with machine learning techniques.

\subsection{Flows for the Neural Network MLT}

1. Input the dataset

2. Perform the process of classification or clustering by evaluating the performance in cycles.

3. Perform 9:1 cross validation on the data set.

4. Define the number of hidden layers for the neural network.

5. Train the Network.

6. Analyze the performance of the model using root means square error, mean square error.

7. Retrain by changing the epochs

\subsection{Flows for NNPO}

In this model the process is similar as described above but in cycles we try to optimize the learning factor and learning momentum. The learning factor define the capability of the model to learn and learning momentum defines the rate with which model adapts to new environment.

\subsection{Flows for FLNN}

1. Input the Dataset.

2. Define the training and target data set

3. Perform sub clustering to generate the cluster centers range for the attributes.

4. Define the membership functions for output and input.

5. Generate the rules

6. Perform defuzzification

7. Performance analysis by testing the model with training data and testing data.

\subsection{Flows for Decision Tree}

1. Input the Dataset.

2. Define the class label in the dataset.

3. Check to see if the dataset type in numeric, if yes converts it to nominal type.

4. Perform Normalization on the dataset.

5. Discreteize of the dataset based on frequency.

6. Apply the cross validation process with training and testing dataset.

7. Apply the Decision tree model to evaluate the gini impurity, information gain.
8. Evaluate the performance of the model.

\subsection{Flows for Naïve Bayes Network}

1. Input the dataset

2. Perform 9:1 split validation

3. Generate the classes based on training dataset.

4. Test the classes on the testing dataset.

5. Evaluate the classification using class precision percentage

6. Remove the classes with less than minimum threshold.

7. Repeat the process.

8. Analyze the performance of the model.

\subsection{Flows for Linear Regression}

1. Retrieve the dataset

2. Identify the class label attribute

3. Convert all the attributes to numeric data types.

4. Normalize the data set

5. Perform linear regression in matlab with parallel testing in cycles

6. A linear regression model provides weights of the attributes to predict the class label which will enhance the process in next cycle.

7. Analyze the performance.

\subsection{Flows of Perceptron}

1. Retrieve the dataset

2. Normalize the datase

3. Discreteize the dataset based on frequency, binning etc.

4. Polynominal to binominal classification with parallel perceptron learning

5. The weight of the attributes generated marks their influence over the "predict label "attribute.

6. Weights can be used to train other MLT's.

7. Analyze the performance.

\section{RESULT AND ANALYSIS}

The software cost estimation was made using different machine learning techniques and the evaluation criteria used was root means square error and square error. The error is defined as the difference between the quantity to be estimated and the actual value. The difference occurs due to randomness or the estimator does not take into account factors that could produce more accurate estimates. Root Means Square Error: RMSE is a meaningful measure of error as it is a measure of variability of the difference. [2] More technically we can say, it states the spread of $y$ values over the predicted $y$ value Square error is defined as the squared value of the root means square error.

$$
R M S E=\frac{\sqrt{\sum_{i=1}^{m}(p y i-y i)^{2}}}{\sqrt[2]{m}}
$$

$\mathrm{m}$ : total \# observations, $\mathrm{py}_{\mathrm{i}}$ : predicted $\mathrm{y}$ value of ith observation and $\mathrm{y}_{\mathrm{i}}$ : actual $\mathrm{y}$ value of the ith observations. 


$$
S E=\frac{\sum_{i=1}^{m}(p y i-y i)^{2}}{m}
$$

The square error shows more precise error in prediction than RMSE for values with +/- 0.0 error range.

Table 1. Example Dataset

\begin{tabular}{|l|l|l|l|l|l|}
\hline Cost Drivers & $\begin{array}{l}\text { Value of } \\
\text { past } \\
\text { project }\end{array}$ & $\begin{array}{l}\text { Numeri } \\
\text { c Value }\end{array}$ & $\begin{array}{l}\text { Value of } \\
\text { Present } \\
\text { project }\end{array}$ & $\begin{array}{l}\text { Numeric } \\
\text { Value }\end{array}$ & $\begin{array}{l}\text { Software } \\
\text { Cost (in \$) }\end{array}$ \\
\hline Size of Application & Low & 0.92 & High & 1.10 & 11956.52 \\
\hline Complexity of Project & Nominal & 1.00 & High & 1.14 & 13630.43 \\
\hline Virtual Machine Volatility & Nominal & 1.00 & Low & 0.87 & 11858.48 \\
\hline Memory Constraints & Nominal & 1.00 & High & 1.07 & 12688.57 \\
\hline Application Experience & High & 0.91 & Nominal & 1.00 & 13943.49 \\
\hline $\begin{array}{l}\text { Run time Performance } \\
\text { Constraint }\end{array}$ & High & 1.11 & High & 1.29 & 16204.59 \\
\hline $\begin{array}{l}\text { Required Development } \\
\text { Schedule }\end{array}$ & Nominal & 1.00 & High & 1.05 & 17014.82 \\
\hline Analyst Capability & Low & 1.15 & High & 0.88 & 13020.04 \\
\hline Virtual Machine Experience & Very Low & 1.22 & Nominal & 1.00 & 10672.16 \\
\hline $\begin{array}{l}\text { Programming Lang } \\
\text { Experience }\end{array}$ & Low & 1.09 & Nominal & 1.00 & 9790.97 \\
\hline $\begin{array}{l}\text { Modem Programming } \\
\text { Practice }\end{array}$ & Low & 1.09 & Low & 1.20 & 10779.05 \\
\hline Use of Software Tools & Low & 1.09 & Nominal & 1.00 & 9889.04 \\
\hline Programmer Capability & Low & 1.14 & High & 1.00 & 8674.6 \\
\hline
\end{tabular}

Table 2. Normalized Form of Dataset PP (Past Project), PrP (Present Project) CD (COST DRIVER)

\begin{tabular}{|c|c|c|}
\hline PP_CD & PrP_CD & Norm_Cost \\
\hline 0.0 & 0.5 & 0.4 \\
\hline 0.3 & 0.6 & 0.6 \\
\hline 0.3 & 0.0 & 0.4 \\
\hline 0.3 & 0.5 & 0.5 \\
\hline 0.0 & 0.3 & 0.6 \\
\hline 0.6 & 1.0 & 0.9 \\
\hline 0.3 & 0.4 & 1.0 \\
\hline 0.8 & 0.0 & 0.5 \\
\hline 1.0 & 0.3 & 0.2 \\
\hline 0.6 & 0.3 & 0.1 \\
\hline 0.6 & 0.8 & 0.3 \\
\hline 0.6 & 0.3 & 0.1 \\
\hline 0.7 & 0.3 & 0.0 \\
\hline
\end{tabular}

\subsection{Neural Network and NNPO}

Input

Hidden 1

Output

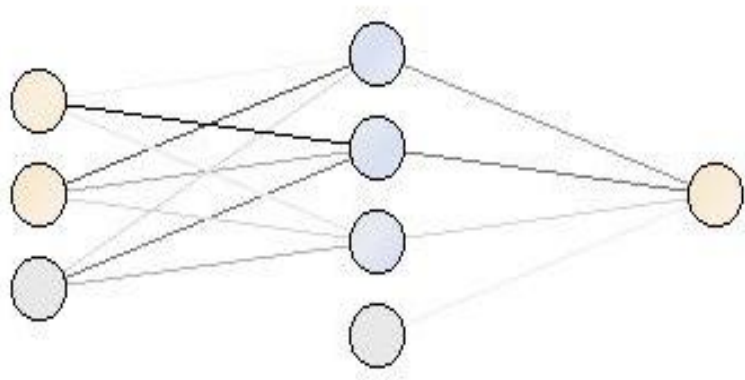

Fig 2: Neural network structure for software project

The input defines the input attributes that are cost drivers of past and present project and actual software cost. The output defines the predicted software cost. The Neural network required 1 hidden layer, 4 hidden neurons and a total of 8 neurons for complete cost estimation. The RMSE for the neural net for the software project was found to be $(0.099$, 0.119 ) and when we performed parameter optimization taking learning rate and learning momentum as the parameter to be optimized we see a significant change in the RMSE values that is for NNPO RMSE is 0.071. Optimized values for learning rate of $\mathrm{NN}$ are 0.7 and learning momentum is 0.1 shows that $\mathrm{NN}$ when trained using above input values, error in estimation is low. Similarly using calculated hidden layer for NN has improved the results of NN

$$
\begin{gathered}
\text { Hidden layers: } \# \text { rows }<\frac{2}{3} \# \text { rows }+\# \text { rows }<2 * \\
\text { \# rows. }(14)
\end{gathered}
$$

When the Neural Net is trained with hidden layers in the range (14) the accuracy is improved and $\mathrm{NN}$ is trained with low training error. 


\subsection{Fuzzy logic trained NN (FLNN)}

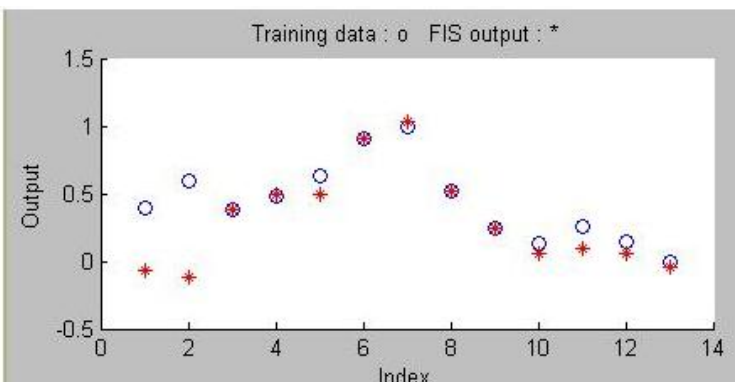

Fig 3: FLNN graph showing mapping of FIS output on training data

The training data is the actual cost and the FIS output is the predicted cost using fuzzy logic. The close mapping of values shows that NN is better trained using Fuzzy logic. The RMSE error of FLNN is 0.136 which shows much improvement over Decision Tree, Naïve Bayes and Perceptron. . A membership function $\mu(\mathrm{X})$ describes the membership of the elements $\mathrm{x}$ of the base set $\mathrm{X}$ in the fuzzy set A. A membership function is defined for those sets of data where precise classification is not possible; hence membership domain is defined based on cluster centers [6]. The "index" on the $\mathrm{x}$ axis is the Epoch rate to train the neural network. The membership function graph is Gaussian type depicting 4 cluster centers ( 2 for each input attribute) generated from the software project dataset

\subsection{Decision Tree}

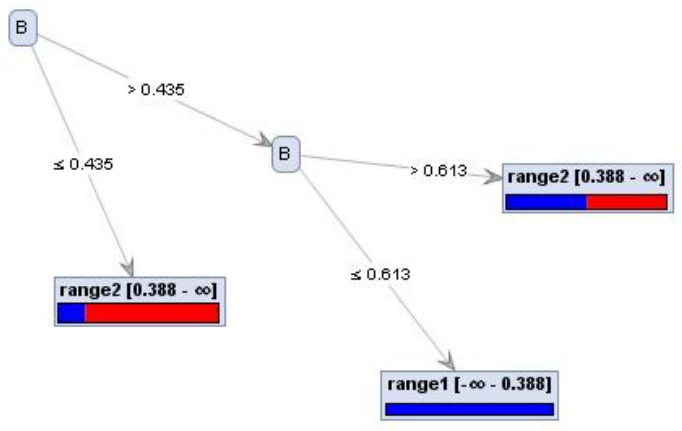

Fig 4: A decision tree classifies the dataset, root node as past project data and leaves as present project

The Decision tree Network is a classification model like NN used to classify the data in the dataset. The dataset before being operated by the decision tree have to be converted into nominal data set so that they can be classified based on yes/no questions [11]. The leaves of the tree show the class label and the splitting attribute taken was past projects data as the information gain was high. The decision tree can be used to derive gini impurity and information gain [11]. Our classification is based on information gain output parameter of the decision tree. The correlation in the dataset is also brought out by the decision tree and the correlation correction can be analyzed using apply model and performance analysis done using rapid miner. The performance of Decision tree was found worse as compared to other machine learning model this is due to lack of understanding the heterogeneous and non-linear data in software cost modeling.

\subsection{Naïve Bayes Classifier (NBC)}

Table 3.Naive Bayes Confusion Matrix

\begin{tabular}{|c|c|c|c|}
\hline & True range & True range & Class \\
$\mathbf{1}$ & 7 & 4 & 63.54 \\
\hline $\begin{array}{c}\text { Pred. range } \\
\mathbf{1}\end{array}$ & & & \\
\hline $\begin{array}{c}\text { Pred. range } \\
\mathbf{2}\end{array}$ & 1 & 1 & 50.00 \\
\hline Class recall & 87.50 & 20.00 & \\
\hline
\end{tabular}

Naïve bayes classifier classifies the information based on Bayesian probability as shown in figure 6. A Bayesian probability is considered to be an evidential probability in which evidences that is cost drivers and actual software cost data is analyzed to predict the cost. The Bayesian theorem is an extension of propositional logic (true/false) that enable to reason uncertainty which exists in $\mathrm{S} / \mathrm{W}$ cost [8]. In naïve bayes classifier, hypothesis is created which is renewed in the light of new, relevant data. The true range defined in the table is the actual value. The prediction range is defined as

(Pred. Range 1)Negative $(\infty, 0.500]$ and (Pred. range 2) Positive $(\infty, 0.500]$. A recall of $20 \%$ defines lower rate of irrelevant instances. Accuracy of the result achieved is $62 \%$.

\subsection{Linear Regression (LR)}

Linear regression is another machine learning technique used in SCE. The RMSE for linear regression shows much improvement than NN, naïve bayes classifier, FLNN. The linear regression uses the concept of curve fitting using linear equation intercept form. LR technique finds its application in forecasting, prediction. Given a variable y and a number of variables $\mathrm{X}_{1}, \mathrm{X}_{2}, \mathrm{X}_{3} \ldots \mathrm{X}_{\mathrm{n}}$ that may be related to $\mathrm{y} L R$ technique can be applied to deduce the relationship between $\mathrm{X}_{\mathrm{i}}$ and $\mathrm{y}$. This relationship is shown in terms of weights. $\mathrm{P}$ values $<0.05$ define acceptable discrimination of the dataset. Greedy algorithm did not provide promising results for Past project driver and worked correctly only for present project cost driver.

Based on M5 prime algorithm Past project has high influence then present project.

Table 4.Linear Regression Results

\begin{tabular}{|l|l|l|l|l|}
\hline $\begin{array}{l}\text { Feature } \\
\text { selection }\end{array}$ & $\begin{array}{l}\text { LR } \\
\text { coefficient }\end{array}$ & $\begin{array}{l}\text { Std. } \\
\text { error }\end{array}$ & $\begin{array}{l}\text { T test } \\
\text { statistics }\end{array}$ & $\begin{array}{l}\text { p- } \\
\text { value }\end{array}$ \\
\hline $\begin{array}{l}\text { M5 (PrP) } \\
\text { primes }\end{array}$ & 0.341 & 0.495 & 0.688 & 0.052 \\
\hline Greedy & 0.368 & 0.362 & 1.016 & 0.048 \\
\hline $\begin{array}{l}\text { M5 } \\
\text { (PaP)prime }\end{array}$ & -0.358 & 0.481 & -0.744 & 0.048 \\
\hline
\end{tabular}

\subsection{Perceptron in SCE}

A perceptron is also a classifying network used in software cost estimation. The main objective of perceptron is classifying the information and assigns weight to the attributes in each classified range [2]. The weight is nothing but the relationship between the input attribute and output attribute. The relationship can be used to train the neural net when coded in MATLAB or perceptron can itself be used to predict software after normalization of the data set. 


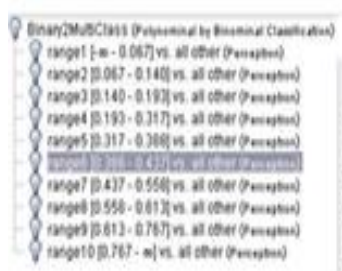

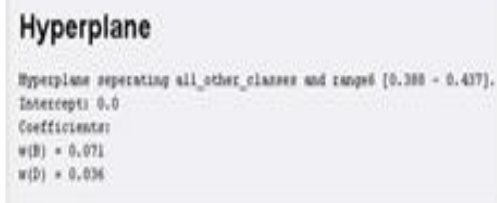

Fig 5: Perceptron trained NN and weights of attributes for each classification

Present project cost drivers have high weights as compared to past project as demonstrated by perceptron.

\subsection{Association Rules Mining}

Table 5. Association Rules: ITEMSET $1 \rightarrow$ ITEMSET 2

\begin{tabular}{|l|l|l|l|}
\hline Itemset 1 & Itemset 2 & Support & Confidence \\
\hline $\begin{array}{l}\text { Past Project } \\
\text { cost Driver }\end{array}$ & $\begin{array}{l}\text { Present } \\
\text { Project cost } \\
\text { Drivers }\end{array}$ & 0.846 & 0.912 \\
\hline $\begin{array}{l}\text { Present } \\
\text { Project cost } \\
\text { Drivers }\end{array}$ & $\begin{array}{l}\text { Past Project } \\
\text { cost Driver }\end{array}$ & 0.634 & 0.772 \\
\hline
\end{tabular}

Association Rules is a rule induction technique of data mining applied to software engineering to generate rules that is correlation in data set to determine support and confidence. The support of an item set $\mathrm{X}$ is defined as the proportion of transaction in the dataset which contain the item set. Confidence of a rule is defined as:

$\operatorname{Conf}(X \rightarrow Y)=\frac{\operatorname{supp}(X \cup Y)}{\operatorname{supp}(X)}$

The result of association rule mining in software project can be applied after discretization by frequency, FP item set and then association rule mining is performed. Form the figure 6 it is clear that for software cost estimation past projects have high influence factor then the present project. On careful simulation we obtained confidence of 0.9 when past project data was taken as label and confidence of 0.7 when present data was taken as label. This conclusion gets stronger in the consequent sub-section of cost prediction based on past project data and present project data.

\subsection{Graphical representation of predicted cost}

We have carried out the research of comparing the machine learning models in software cost estimation. We collected cost drivers values used in past projects and cost drivers values to be used in present projects. The present projects are similar in requirements to past project with variations in behavior and quality. For example: UI used in past project was command prompt based whereas in new UI it is application based, so the cost driver rose from low to high in new project. Similar characteristics were taken into consideration to predict the cost based on available data.

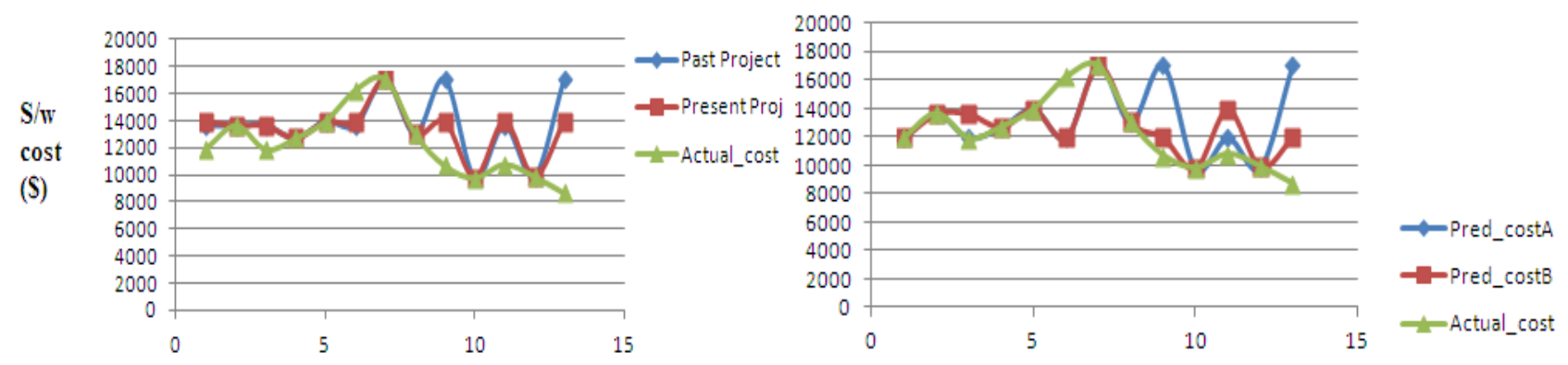

Fig 6: software cost predicted using Naïve bayes and Decision Tree respectively
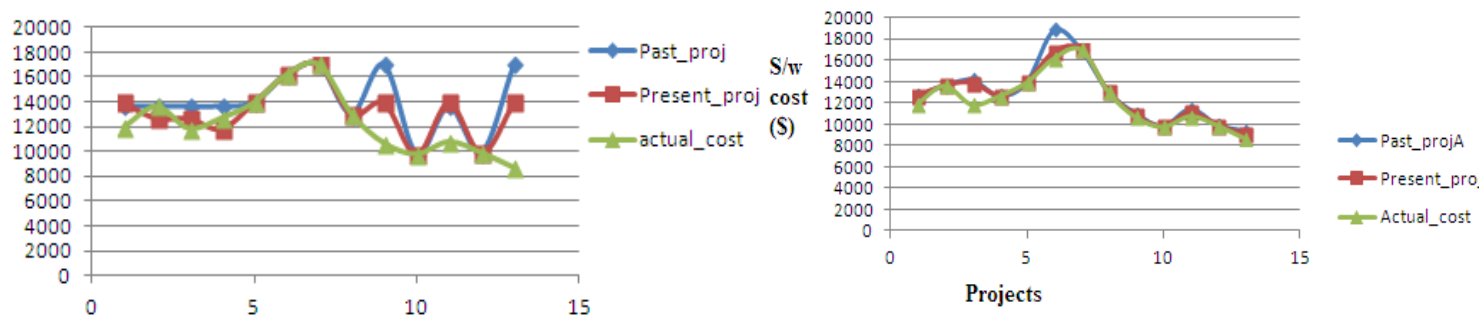

Fig 7: software cost predicted using Perceptron and Neural Network PO respectively 


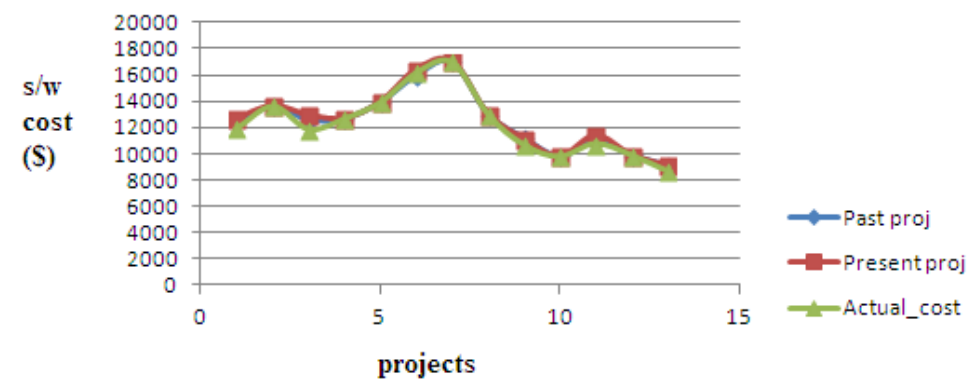

Fig 8: software cost predicted using Linear Regression

Table 6. Comparison Between Past and Present Cost Drivers using Machine Learning Techniques

\begin{tabular}{|l|l|l|l|}
\hline S.no & Machine Learning Model & $\begin{array}{l}\text { Performance Past Cost } \\
\text { Drivers }\end{array}$ & $\begin{array}{l}\text { Performance Present Cost } \\
\text { Drivers }\end{array}$ \\
\hline 1. & Neural Network & RMSE:0.099 SE:0.013 & $\begin{array}{l}\text { RMSE:0.119 } \\
\text { SE: } 0.097\end{array}$ \\
\hline 2. & $\begin{array}{l}\text { Neural Network with Parameter } \\
\text { optimization }\end{array}$ & $\begin{array}{l}\text { RMSE:0.071 } \\
\text { SE: } 0.008\end{array}$ & $\begin{array}{l}\text { RMSE: } 0.09 \\
\text { SE: } 0.011\end{array}$ \\
\hline 3. & Fuzzy Logic Neural Network & RMSE:0.136 SE:0.110 & $\begin{array}{l}\text { RMSE: } 0.142 \\
\text { SE: } 0.116\end{array}$ \\
\hline 4. & Decision Tree & $\begin{array}{l}\text { RMSE: } 0.307 \\
\text { SE: } 0.194\end{array}$ & $\begin{array}{l}\text { RMSE:0.376 } \\
\text { SE: } 0.204\end{array}$ \\
\hline 5. & Naïve Bayes Classifier & RMSE:0.186 SE:0.107 & $\begin{array}{l}\text { RMSE: } 0.211 \\
\text { SE: } 0.126\end{array}$ \\
\hline 6. & Perceptron & RMSE:0.148 SE:0.114 & $\begin{array}{l}\text { RMSE:0.201 } \\
\text { SE: } 0.119\end{array}$ \\
\hline 7. & Linear Regression technique & $\begin{array}{l}\text { RMSE:0.09 } \\
\text { SE: } 0.01\end{array}$ & $\begin{array}{l}\text { RMSE: } 0.108 \\
\text { SE: } 0.017\end{array}$ \\
\hline
\end{tabular}

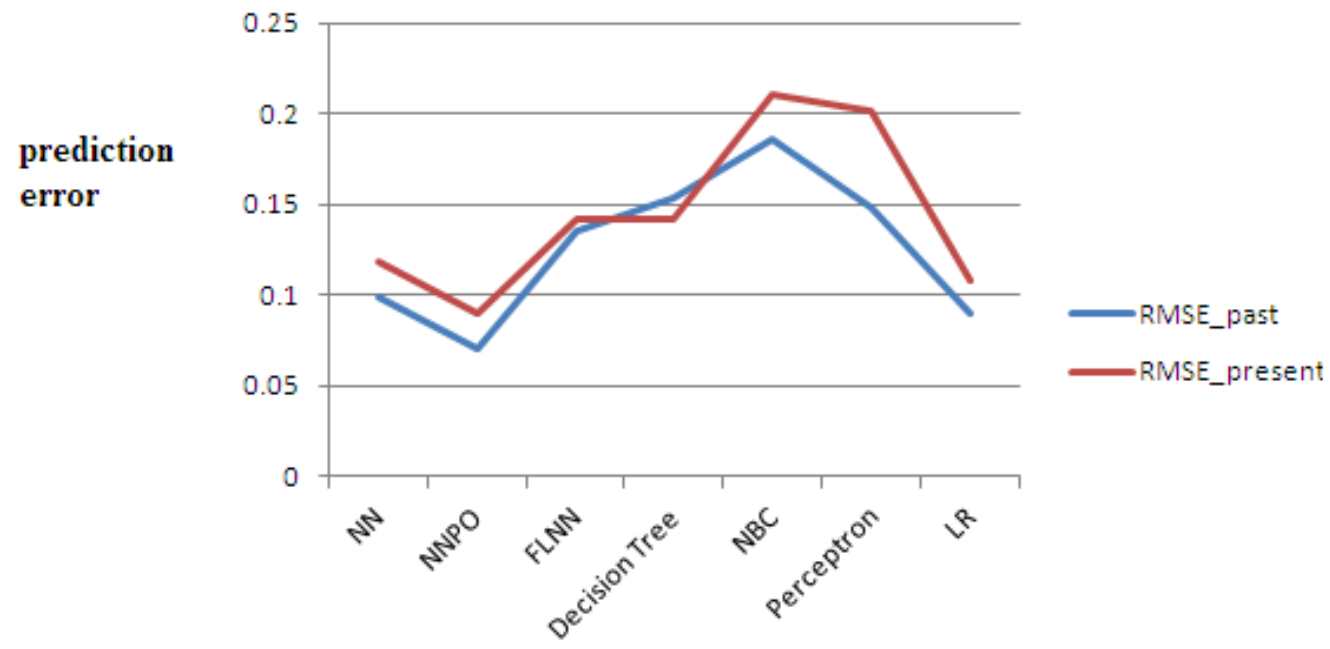

Fig 9: Error in prediction when Model was used on the dataset (RMSE values)

\section{CONCLUSION AND FUTURE WORK}

Some of the software projects in organizations suffer from over budget or failure. This happens due to inaccurate measures to estimate the costs. The algorithmic models were foundation for the estimation but there considered the current project to estimate, considering today's development environment we emphasize on re-engineering or re-use engineering. So we need those estimation models which can predict the cost from historical data. Estimation using machine learning technique focuses on prediction based on rules generated from the training data, back propagation neural network with updates the weights which signifies the importance of each attribute, curve fitting based prediction. Our research focuses on finding one model that provides least prediction error when applied to project, irrespective of their class. We considered a neural network, tried to improve it by optimization of parameters, generated fuzzy rules to train neural network, naïve bayes network classifier which detect 
the class based on true range and class precision, linear regression and perceptron model that was used to enhance the capability of NN. Based on the performance result obtained for various machine learning technique neural network with parameter optimization MLT was proved to be a versatile technique is software cost estimation with minimum Root Mean Sq. error (RMSE).

More similar type of studies must be carried out with large projects will different attributes to get better accuracy. Scale factors can also be used for estimation. In new generation software development we have TCP based GUI, Plug in based GUI and Application based GUI, so we can try to develop cost drivers values for each subclass attributes so as to get more detailed estimation and performance of the models can be well tested.

\section{REFERENCES}

[1] Venkatachalam, A.R 1993 Software Cost Estimation Using Artificial Neural Networks, Proceedings of International Joint Conference on Neural Network, pp. 987-990.

[2] Samson, Bill 1997 Software cost estimation using an Albus perceptron (CMAC), Information and Software technology ELSEVIER, pp. 55-60,1997.

[3] Boehm, B.W, 1981 Software Engineering Economics, Software Engineering Books, Prentice Hall.

[4] Krishnamurthy, S. and Fisher, D. 1995 Machine Learning Approaches to Estimating Software Development Effort, IEEE transaction on Software Engineering, pp. 126-137.
[5] Malhotra, Ruchika, Kaur, Arvinder and Singh, Yogesh 2010 Machine Learning Methods for Software Effort Prediction, ACM SIGSOFT Software Engineering Notes, pp. 1-6.

[6] Attarzadeh, Iman and Hock Ow, Siew, 2011 Improving Estimation Accuracy of the COCOMO II Using an Adaptive Fuzzy Logic Model, IEEE International Conference on Fuzzy Systems, pp. 2458-2464.

[7] Agarwal, K.K and Singh, Yogesh, 2005 Software Engineering, Software Engineering Books, New Age International Publishers.

[8] Rish, I, 2001 An empirical study of naïve Bayes classifier, IJCAI workshop on empirical methods in artificial intelligence, pp. 41-46.

[9] Kabir, Md Faisal, 2011 Enhanced Classification Accuracy on Naïve Bayes Data Mining Models, International Journal of Computer Application, pp. 9-16.

[10] Idri, Ali and Elyassami, Sanaa , 2011 Applying Fuzzy ID3 Decision Tree for Software Effort Estimation, International Journal of Computer Science Issues, pp. 131-138.

[11]Baskeles, Bilge, 2007 Software Effort Estimation Using Machine Learning Methods, IEEE $22^{\text {nd }}$ Symposium on Computer and Information Sciences, pp. 1-6.

[12] Papatheocharous, Efi, 2012 Software Cost Modelling and Estimation Using Artificial Neural Network Enhanced Input Sensitivity Analysis, Journal of Universal Computer Science, pp. 1-30. 